HIV

\title{
The effects of urethritis on seminal plasma HIV-1 RNA loads in homosexual men not receiving antiretroviral therapy
}

\author{
S T Sadiq, S Taylor, A J Copas, J Bennett, S Kaye, S M Drake, S Kirk, D Pillay, I V D Weller
}

Sex Transm Infect 2005;81:120-123. doi: 10.1136/sti.2004.010249

See end of article for authors' affiliations

....................

Correspondence to: Dr Tariq Sadiq, HIV/ GUM, Department of Cellular and Molecular Medicine, St George's Hospital Medical School, Cranmer Terrace, London SW17 ORE, UK; ssadiq@ sghms.ac.uk

Accepted for publication 5 July 2004

\begin{abstract}
Objectives: To examine the effects of urethritis and its treatment on semen plasma HIV-1 RNA load in HIV-1 infected men not receiving antiretroviral therapy (ART), in a developed world setting.

Methods: Prospective case-control study. HIV-1 infected homosexual men, not receiving ART for at least 3 months, with (cases) and without (controls) symptomatic urethritis, were recruited. Blood and semen were collected for HIV-1 RNA quantification at presentation, before antibiotic therapy, and at 1 and 2 weeks.

Results: 20 cases (13 gonococcal urethritis and/or chlamydial urethritis (GU/CU) and seven non-specific urethritis (NSU)) and 35 controls were recruited. Baseline characteristics and blood plasma viral load were similar in cases and controls. Mean log semen plasma viral loads were higher among those with GU/CU compared with controls ( 4.27 log versus 3.55 log respectively; $p=0.01$ ) but not in those with NSU (3.48 $\log ; \mathrm{p}=0.82$ ). Following antibiotics, semen plasma viral loads fell by a mean of $0.25 \log (95 \% \mathrm{Cl}: 0.03$ to 0.47 ) in those with GU/CU. Semen plasma viral loads did not fall in those with NSU.

Conclusions: In this study of 55 homosexual men not on ART, semen plasma viral loads were approximately fivefold higher in those with GU/CU, but not NSU, compared with controls. Treatment of GU/CU resulted in reduction in semen plasma viral loads. Although absolute effects were considerably lower when compared to patients from a similar study from sub-Saharan Africa, our data demonstrate the potential for sexually transmitted infections to enhance HIV infectivity of men not receiving ART in the developed world.
\end{abstract}

$M$ ost HIV-1 infections in adults worldwide occur sexually, and there is biological and epidemiological evidence that the quantity of virus in genital secretions is an important determinant of transmission. ${ }^{1-8}$ We have recently demonstrated no effect of sexually transmitted infections (STIs) on seminal plasma viral loads (SPVL) in homosexual men on fully suppressive antiretroviral therapy (ART) living in the United Kingdom. ${ }^{9}$ Among those not on ART, urethritis has been associated with increased genital shedding of HIV-l in studies from subSaharan Africa, with differences of over 100000 copies $/ \mathrm{ml}$ in median SPVL observed in cases of gonococcal urethritis (GU) compared to those without STIs in one study. ${ }^{10}{ }^{11}$ In the developed world, a limited number of small studies have suggested that STIs may increase SPVL in those not taking ART. ${ }^{12} 13$

It is possible that effects of STIs on SPVLs, observed in Africa, may be greater than those observed in the developed world. HIV-1 infected individuals in Africa have, on average, higher blood plasma viral loads (BPVL) and states of immune activation than those in the developed world ${ }^{14}{ }^{15}$ and the effects of inflammatory cytokines on viral replication may be greater on prevalent subtypes in Africa compared to subtype $\mathrm{B}$, found more commonly in Europe and North America. ${ }^{16}$ Furthermore, delayed health seeking behaviour of those with STIs in the developing world may allow STIs to have greater impact before treatment is given. ${ }^{17}$ We studied the effects of urethritis on SPVL in homosexual men with HIV infection who were not on ART.

\section{METHODS}

HIV-1 infected homosexual men not receiving ART for at least 3 months attending two UK sexual health clinics either with urethritis (cases) or for a sexual health check up but with no STI (controls) were recruited prospectively between
November 2000 and October 2002 (study visit 1). Patients were excluded if they had an episode of urethritis or systemic illness in the previous month. Participants had routine urethral swabs for gonorrhoea (by microscopy and culture), chlamydia (using ligase chain reaction; Abbott Diagnostics, Abbot Park, IL, USA), and non-gonococcal urethritis (NGU) by microscopy. For the study, in those who were negative for gonorrhoea on microscopy, NGU was defined as those patients symptomatic for urethritis with five or more polymorphs per high power field $(\mathrm{p} / \mathrm{hpf}$ ) on microscopy or those asymptomatic patients with $10 \mathrm{p} / \mathrm{hpf}$ or more on microscopy. This definition was used because of our observation that consistency of microscopy alone for predicting NGU is poor when polymorph counts are low. ${ }^{18}$ The initial diagnosis of NGU was changed to non-specific urethritis (NSU) if subsequent chlamydia and gonococcal culture tests remained negative. In addition to serological tests for syphilis taken on their first visit, blood was also collected for HIV-1 RNA quantification and patients then provided a semen sample by masturbation into a sterile container. All patients provided semen samples before voiding urine and were advised against using lubricants during masturbation.

Patients diagnosed with urethritis, whether GU or NGU, were treated with appropriate antibiotics. Cases and controls were asked to attend the following week (visit 2) and 2 weeks later (visit 3) for repeat smears, gonococcal culture, and blood and semen samples. At all visits, clinical and demographic data were collected including sexual histories.

\footnotetext{
Abbreviations: ART, antiretroviral therapy; BPVL, blood plasma viral loads; CU, chlamydial urethritis; GEE, generalised estimating equations; GU, gonococcal urethritis; NGU, non-gonococcal urethritis; NSU, nonspecific urethritis; PCR, polymerase chain reaction; $\mathrm{p} / \mathrm{hpf}$, polymorphs per high power field; SPVL, seminal plasma viral loads
} 
A sample size of 20 cases and controls was required to give approximately $80 \%$ power to detect as significant a difference in mean log-SPVL at first visit of 0.7 (that is, a fivefold difference in SPVL), as observed previously in Africa, relative to a standard deviation of measurements in each group of 0.8 , and taking the standard 5\% significance level. It was decided to try to recruit more controls to increase this power.

\section{Virology methods}

Semen and blood samples were centrifuged within 2 hours of collection and the plasma and cellular components stored at $-70^{\circ} \mathrm{C}$. HIV-1 RNA was extracted from blood and semen plasma by a silica gel capture method previously observed to successfully remove inhibitors of the polymerase chain reaction $(\mathrm{PCR})^{19}$ and quantified using an in-house, internally calibrated reverse transcribed PCR assay (RT-QPCR, Department of Virology UCL, London). The lower limit of quantification was 1000 copies/ml.

\section{Statistical methods}

Cases were compared with controls with respect to age, years since HIV diagnosis, ethnicity, median numbers of partners in previous 3 months, and most recent CD4 count and HIV-1 viral load before first visit. For comparisons of age, number of partners, CD4 count and time since HIV diagnosis the MannWhitney test was used. For viral loads before first visit, and also at first visit, the $t$ test was used. In all analysis of HIV-1 RNA loads undetectable measurements were considered as 500 copies/ml (half the limit of detection), and $\log _{10}$ values were used. To compare ethnicity and HIV-1 RNA detectability at visit 1 Fisher's exact test was used. To compare HIV-1 RNA loads in blood and semen within patients at visit 1 the paired $t$ test was used, and their correlation assessed using Pearson's correlation coefficient. Average changes in HIV-1 RNA loads across study visits were estimated for cases and controls, and these changes compared. This analysis was based on generalised estimating equations (GEE) of Stata 7, because of multiple measurements for patients, selecting an exchangeable working correlation structure, and using the robust standard errors. As planned subgroup analysis, comparisons with controls were made for all cases, for NSU cases alone, and cases with chlamydial urethritis (CU) or GU.

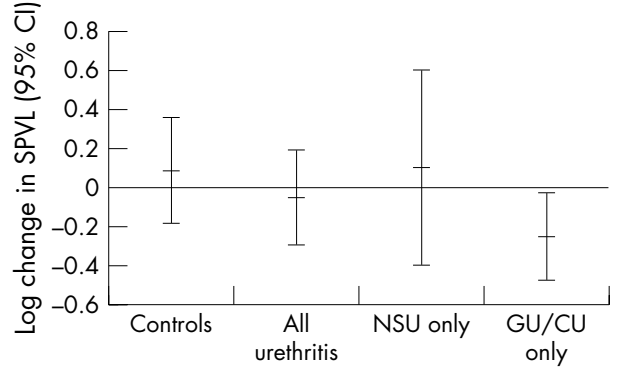

Figure 1 Mean $\log _{10}$ change in semen plasma viral load from visit 1 to follow up. $\mathrm{GU} / \mathrm{CU}=$ gonococcal or chlamydial urethritis; NSU = nonspecific urethritis (non-gonococcal and non-chlamydial). Level of detection $=1000$ ( $3 \mathrm{log})$, and undetectable viral loads considered as 500 $(2.7 \mathrm{log})$. Mean change in patients with $\mathrm{GU} / \mathrm{CU}=-0.25 \log (-0.47$ to $0.03) p=0.028$.

\section{RESULTS}

Twenty cases (nine GU, three CU, one combined CU and GU, and seven NSU) and 35 controls were recruited. In this study, all cases had polymorph counts of $>10 \mathrm{p} / \mathrm{hpf}$ counts and all controls counts of $<5 \mathrm{p} / \mathrm{hpf}$. All cases were symptomatic, except one with NSU who had a polymorph count of $11 \mathrm{p} /$ hpf. Three of the remaining NSU cases had polymorph counts of between 10 and $20 \mathrm{p} / \mathrm{hpf}$ and the other three, counts of $>20 \mathrm{p} / \mathrm{hpf}$. All cases of CU or GU had polymorph counts of $>20 \mathrm{p} / \mathrm{hpf}$ except one with GU with a count of $15 \mathrm{p} / \mathrm{hpf}$. Seven controls had symptoms of urethral discomfort, but were negative for chlamydia and gonorrhoea. One case with GU and two controls were receiving antibiotics for unrelated minor infections at presentation. Median age, years since HIV diagnosis, ethnicity, numbers of sexual partners in the previous 3 months, pre-study BPVL, and pre-study CD4 count were similar between cases and controls (table 1).

\section{BPVLs and SPVLs at study visit 1 and follow up (see table 1)}

HIV-1 RNA was detectable in 16/20 cases compared with $23 / 35$ controls in semen ( $p=0.36$, Fisher's exact test $)$ and in $18 / 20$ cases compared with $33 / 35$ controls in blood $(p=0.62)$.

Table 1 Baseline characteristics and viral loads of cases and controls

\begin{tabular}{|c|c|c|c|}
\hline & Urethritis & Controls & P Value ${ }^{* *}$ \\
\hline Number & 20 (9 GU, $1 \mathrm{GU} / \mathrm{CU}, 3 \mathrm{CU}, 7 \mathrm{NSU})$ & 35 & - \\
\hline Median age (years) (range) & $33.3(23.9-48.5)$ & $35.6(24.5-61.8)$ & 0.345 \\
\hline Years since HIV diagnosis (range) & $3.36(0.41-14.18)$ & $1.13(0.11-16.19)$ & 0.069 \\
\hline White ethnicity (n) & 18 & 31 & 1.00 \\
\hline Median partners in last 3 months (range) & $4(1-21)$ & $3(0-51)$ & 0.297 \\
\hline Mean pre-study $\log _{10}$ BPVL $(95 \% \mathrm{CI})$ & 4.26 (3.90 to 4.62$)$ & 4.34 (4.06 to 4.63$)$ & 0.728 \\
\hline Median pre-study CD4 count (range) & $475(56-1220)$ & $477(44-1590)$ & 0.937 \\
\hline \multicolumn{4}{|l|}{ BPVL at study visit 1} \\
\hline All urethritis & 4.11 (3.76 to 4.45$)$ & $4.21(4.03$ to 4.40$)$ & 0.550 \\
\hline GU/CU only & 4.17 (3.75 to 4.59$)$ & - & 0.821 \\
\hline NSU only & 4.00 (3.22 to 4.77$)$ & - & 0.385 \\
\hline \multicolumn{4}{|l|}{ BPVL after study visit 1} \\
\hline All urethritis & 4.19 (3.78 to 4.59$)\left(n=22 / 15^{*}\right)$ & $4.27(4.02$ to 4.52$)\left(n=23 / 21^{*}\right)$ & 0.752 \\
\hline GU/CU only & $4.38(3.90$ to 4.87$)\left(n=13 / 9^{*}\right)$ & 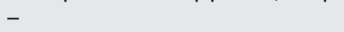 & 0.657 \\
\hline NSU only & $3.92(3.24$ to 4.60$)\left(n=9 / 6^{*}\right)$ & - & 0.306 \\
\hline \multicolumn{4}{|l|}{ SPVL at study visit 1} \\
\hline All urethritis & 3.99 (3.53 to 4.45 ) & 3.55 (3.27 to 3.83 ) & 0.078 \\
\hline GU/CU only & 4.27 (3.66 to 4.87$)$ & - & 0.014 \\
\hline NSU only & $3.48(2.78$ to 4.17$)$ & - & 0.820 \\
\hline \multicolumn{4}{|l|}{ SPVL after study visit 1} \\
\hline All urethritis & 3.88 (3.54 to 4.23$)\left(n=21 / 16^{*}\right)$ & $3.59(3.24$ to 3.94$)\left(n=24 / 23^{*}\right)$ & 0.228 \\
\hline GU/CU only & $4.12(3.54$ to 4.69$)\left(n=12 / 10^{*}\right)$ & - & 0.111 \\
\hline NSU only & $3.52(3.39$ to 3.66$)\left(n=9 / 6^{*}\right)$ & - & 0.823 \\
\hline
\end{tabular}

GU: gonococcal urethritis; CU: chlamydial urethritis; NSU: non-specific urethritis; BPVL: mean $\log _{10}$ blood plasma viral loads; SPVL: mean log 10 semen plasma viral loads.

*Figures quoted are number of measurements/number of patients: **p value from comparison with controls. 
BPVLs were higher than SPVLs in controls by $0.66 \log$ $(\mathrm{p}<0.001)$ and there was a fairly good correlation between BPVL and SPVL $(r=0.46, \mathrm{p}=0.005$ Pearson coefficient $)$. Among cases overall and in patients with GU or CU, BPVLs were similar to SPVLs $(p=0.58$ and $p=0.52$ respectively, paired $t$ test) and there was again a good correlation between BPVL and SPVL $(r=0.61, \mathrm{p}=0.004$ and $r=0.71, \mathrm{p}=0.006$, respectively). SPVLs appeared to be lower than BPVLs, in those with NSU, by $0.52 \log (p=0.07)$.

There was little difference in mean log BPVL between cases and controls. Compared with controls mean log SPVL appeared higher in cases overall, (3.99 log for cases $v 3.55$ $\log$ for controls; $p=0.08)$, significantly higher in GU/CU cases (4.27 log; $\mathrm{p}=0.014$ ) but were similar in NSU cases (3.48 $\log ; p=0.82) \quad$ (see table 1$)$. Little difference was detected either in SPVL or BPVL in cases of CU compared with GU (mean BPVL: $4.5 \log v 4.07 \log , \mathrm{p}=0.266$; mean SPVL: $4.58 \log v 4.15 \log ; \mathrm{p}=0.44$, respectively).

At follow up 16/24, 6/24, and 1/24 controls and 9/16, 2/16, and 5/16 cases provided semen samples at visit 2 only, visit 3 and at both follow up visits respectively. More specifically among the cases at follow up, semen samples were provided by $6 / 10,2 / 10$, and $2 / 10$ with GU/CU and $3 / 6,0 / 6$, and $3 / 6$ with NSU at visit 2, visit 3 and at both follow up visits respectively. Little difference was detected in mean BPVL or SPVL between cases overall and controls at follow up. Among those with CU/GU, mean SPVL remained approximately half a log higher compared with controls but this difference was not significant.

\section{Changes in log viral loads from visit 1 to follow up (see fig 1)}

No significant changes in BPVL or SPVL from visit 1 to follow up were detected in cases overall, or controls. However, among those with GU/CU alone, SPVLs, but not BPVLs, decreased following antibiotic treatment by on average 0.25 $\log (95 \%$ CI 0.03 to $0.47, \mathrm{p}=0.028)$. When compared with the changes observed among controls, this effect appeared to be broadly maintained with a relative reduction in SPVL in GU/CU cases of $0.34 \log (-0.01$ to $0.68 ; p=0.056)$. Little change in SPVL was observed in those with NSU alone.

\section{DISCUSSION}

This study of $\mathbf{5 5}$ homosexual men is the largest as yet from the developed world examining effects of sexually transmitted infections on seminal plasma viral load in those not on ART. Compared with controls without STIs, SPVLs were approximately fivefold higher in those with GU or CU but were not higher in those with NSU. Additionally, SPVLs were similar to BPVLs among those with GU or CU whereas SPVLs were approximately half a log lower than BPVLs among controls and those with NSU. Treatment of GU or CU resulted in reduction in SPVLs by a small but significant amount over 1 to 2 weeks. Thus, these results indicate GU and CU, though not NSU, increase SPVL in those not on ART.

Previously, similar studies in the developed world have been small and few in number. A case report of a $2 \log$ reduction in SPVL following treatment of $\mathrm{CU}^{12}$ did not comment on changes in BPVL and in a study of four patients not on ART with asymptomatic urethritis, two had high SPVLs but with BPVLs that were also high. ${ }^{13}$ Another study from the United Kingdom showed small but statistically significant decreases in semen HIV-1 proviral load following treatment of three cases of GU and one case of symptomatic NGU. ${ }^{20}$ These changes in proviral load might be expected given the marked, cellular inflammatory response observed in GU and the association between detectability of cell associated virus and semen leucocyte count. ${ }^{8}$ However, increases in both cell free and cell associated HIV-1 in semen are important as both may be transmissible. ${ }^{21}$ In the uninflamed genital tract, though detection of proviral and cell free HIV-1 in semen are associated, ${ }^{22}$ cell free HIV-1 RNA appears phylogenetically distinct from cell associated HIV-1.23 Previous work has suggested that cell free virus in semen is derived locally in the genital tract during urethritis ${ }^{24}$ but it remains unclear whether the increase in HIV-1 RNA in semen during STIs is derived from seminal leucocytes.

In sub-Saharan Africa, urethritis has been associated with increased genital shedding of HIV-1, with median differences of over 100000 copies/ml in SPVL observed in GU cases compared to those without STIs, an approximately fivefold difference. $^{1011}$ This relative effect of GU/CU on SPVLs is similar to those in our study. However, the absolute effect on SPVLs is considerably higher than our study, where the difference in median SPVL between those with GU and controls was only 15000 copies/ml. Explanations for the observed differences between the two settings include patients in the African study more likely to be having late stage HIV disease at presentation (baseline CD4 counts appeared slightly higher in our study), the higher baseline viral loads in blood and semen previously observed in Africa when compared with the developed world and matched for CD4 count, ${ }^{14}$ and the heightened states of immune activation observed there which appear to be environmentally driven. ${ }^{15}$ BPVLs at baseline were higher by up to $1 \log$ in the African study compared with our study.

A probabilistic model of HIV-1 transmission between heterosexuals has been developed from biological and epidemiological data from the United States and Switzerland. ${ }^{25} \mathrm{~A}$ model such as this is unlikely to be completely accurate for homosexual or African men or for the effect of STIs on SPVL. However, crudely applying this model to our data suggests that the HIV male to female per contact transmission probability would increase threefold from approximately one per 1000 to up to three per 1000 during GU or CU. Applying the model similarly to the African data would see an increase of transmission probability from three per 1000 to nine per 1000. It is possible therefore that the effects of these STIs on SPVL may not have as great an impact on transmission risk of HIV-1 in the developed world as in Africa. Clearly, however, more appropriate models and further research on the implications of our findings on HIV-1 transmission are required.

Our work suggests that in the small number of patients with chlamydial infection, the effect on SPVL appeared to be just as pronounced as those with gonorrhoea. This is important as Chlamydia trachomatis is a common cause of urethritis in homosexual men. ${ }^{26}{ }^{27}$ Our findings on NSU may not be surprising given that infection does not always cause this condition. Furthermore, the diagnosis of NSU by microscopy is subject to considerable observer variation ${ }^{18}$ as opposed to the microbiological diagnoses of gonorrhoea by culture or chlamydia by nucleic acid amplification tests, though we did try to limit this variation by restricting asymptomatic cases to higher polymorph counts. Additionally our findings in relation to NSU may not apply to heterosexual men as its aetiology is perhaps different from those in homosexual men. For example, Trichomonas vaginalis, an important cause of urethritis in heterosexuals in some setting ${ }^{28}{ }^{29}$ and associated with increased shedding of HIV-l in semen, ${ }^{28}$ is rare in homosexuals. ${ }^{26}$ Further research with more patients might more rigorously address the issue of how the magnitude of changes in HIV-1 viral load differ between cases of NSU, GU, and CU.

It is important to note that of those who attended for follow up only 7/24 controls and 7/16 cases attended study visit 3 (at 2 weeks after first presentation). Among cases, similar follow up patterns were observed in those with either 
GU/CU or NSU. The African studies ${ }^{10}$ suggest that the maximum reduction of SPVL was seen at 2 weeks after starting antibiotic treatment implying that the $0.25 \log$ reduction in SPVL we observed in GU/CU cases may have been an underestimate.

We previously demonstrated in a separate study that in a group of men similar to those of this study but receiving fully suppressive ART and with GU or CU, SPVLs remained undetectable. ${ }^{9}$ In a small subset of patients in whom virus was not suppressed in blood, high amounts of drug resistant virus were detected in seminal plasma, though in only one case did treatment of gonorrhoea result in reduction of SPVL. ${ }^{930}$ Our current study would thus strengthen the notion that antiviral therapy attenuates effects of STIs on genital shedding of HIV-1. As ART becomes more widely used, these attenuating effects, need to be confirmed in developing world settings because of high rates of STIs there and potential for widespread transmission of drug resistant HIV-1.

This study has demonstrated that gonococcal and chlamydial urethritis among homosexual men in the United Kingdom increases shedding of HIV-1 in semen and treatment of urethritis reduces its shedding. Controlling STIs in HIV-1 infected homosexual men may be critical in controlling the spread of HIV-l among them.

\section{ACKNOWLEDGEMENTS}

The authors acknowledge with gratitude the staff and patients at the Mortimer Market Centre, Camden Primary Care Trust and at the Department of Sexual Medicine, Birmingham Heartlands Hospital.

\section{CONTRIBUTORS}

STS, ST, DP, and IVDW conceived the study; STS wrote the study protocol and together with AJC designed the study; STS, ST, and SMD recruited patients for the study; STS performed viral load analysis and with JB; SKa and SKi validated the semen viral load assay. AJC performed statistical analysis; STS wrote the paper, which was principally reviewed by IVDW and AJC. All authors reviewed and contributed to the final draft.

Ethics approval for this study was received by Camden and Islington Community Health Services local research ethics committee.

\section{Authors' affiliations}

S T Sadiq, A J Copas, I V D Weller, Centre for Sexual Health and HIV Research, Department of Primary Care and Population Sciences, Royal Free and University College Medical School, University College, London, UK

S Taylor, S M Drake, Department of Sexual Medicine, Birmingham Heartlands Hospital, Birmingham, UK

J Bennett, S Kaye, S Kirk, D Pillay, Centre for Virology, Division of Infection and Immunity, Royal Free and University College Medical School, University College, London, UK

S T Sadiq, HIV/GUM, Department of Cellular and Molecular Medicine, St George's Hospital Medical School, London, UK

Funding: Internal funding from UCL.

Conflict of interest: None.

\section{REFERENCES}

1 De Vincenzi I. A longitudinal study of human immunodeficiency virus transmission by heterosexual partners. European Study Group on Heterosexual Transmission of HIV. N Engl J Med 1994;331:341-6.

2 Lee TH, Sakahara N, Fiebig E, et al. Correlation of HIV-1 RNA levels in plasma and heterosexual transmission of HIV-1 from infected transfusion recipients. J Acquir Immune Defic Syndr Human Retrovir 1996:12:427-8.

3 Quinn TC, Wawer MJ, Sewankambo N, et al. Viral load and heterosexual transmission of human immunodeficiency virus type 1. Rakai Project Study Group. N Engl J Med 2000;342:921-9.
4 Coombs RW, Speck CE, Hughes JP, et al. Association between culturable human immunodeficiency virus type 1 (HIV-1) in semen and HIV-1 RNA levels in semen and blood: evidence for compartmentalization of HIV-1 between semen and blood. J Infect Dis 1998;177:320-30.

5 Tachet A, Dulioust E, Salmon D, et al. Detection and quantification of HIV-1 in semen: identification of a subpopulation of men at high potential risk of viral sexual transmission. AIDS 1999;13:823-31.

6 Fiscus SA, Vernazza PL, Gilliam B, et al. Factors associated with changes in HIV shedding in semen. AIDS Res Human Retrovir 1998;14(Suppl 1):S27-S31.

7 Vernazza PL, Gilliam BL, Dyer J, et al. Quantification of HIV in semen: correlation with antiviral treatment and immune status. AIDS 1997; 11:987-93.

8 Xu C, Politch JA, Tucker L, et al. Factors associated with increased levels of human immunodeficiency virus type 1 DNA in semen. J Infect Dis 1997:176:941-947.

9 Sadiq ST, Taylor S, Kaye S, et al. The effects of antiretroviral therapy on HIV-1 RNA loads in seminal plasma in HIV-positive patients with and without urethritis. AIDS 2002; 16:219-25.

10 Cohen MS, Hoffman IF, Royce RA, et al. Reduction of concentration of HIV-1 in semen after treatment of urethritis: implications for prevention of sexual transmission of HIV-1. AIDSCAP Malawi Research Group. Lancet 1997;349:1868-73

11 Moss GB, Overbaugh J, Welch M, et al. Human immunodeficiency virus DNA in urethral secretions in men: association with gonococcal urethritis and CD4 cell depletion. J Infect Dis 1995;172:1469-74.

12 Eron JJ Jr, Gilliam B, Fiscus S, et al. HIV-1 shedding and chlamydial urethritis. JAMA 1996;275:36.

13 Winter AJ, Taylor S, Workman J, et al. Asymptomatic urethritis and detection of HIV-1 RNA in seminal plasma. Sex Transm Infect 1999;75:261-3.

14 Dyer JR, Kazembe P, Vernazza PL, et al. High levels of human immunodeficiency virus type 1 in blood and semen of seropositive men in subSaharan Africa. J Infect Dis 1998;177:1742-6.

15 Clerici $M$, Butto $S$, Lukwiya $M$, et al. Immune activation in africa is environmentally-driven and is associated with upregulation of CCR5. ItalianUgandan AIDS Project. AIDS 2000;14:2083-92.

16 Montano MA, Nixon CP, Ndung'u T, et al. Elevated tumor necrosis factoralpha activation of human immunodeficiency virus type 1 subtype $C$ in Southern Africa is associated with an NF-kappaB enhancer gain-of-function. $J$ Infect Dis 2000;181:76-81.

17 Faxelid E, Ahlberg BM, Ndulo J, et al. Health-seeking behaviour of patients with sexually transmitted diseases in Zambia. East Afr Med J 1998;75:232-6.

18 Smith R, Copas A, Prince M, et al. Poor sensitivity and consistency of microscopy in the diagnosis of low grade non-gonococcal urethritis. Sex Transm Infect 2003;79:487-90.

19 Boom R, Sol CJ, Salimans MM, et al. Rapid and simple method for purification of nucleic acids. J Clin Microbiol 1990;28:495-503.

20 Atkins MC, Carlin EM, Emery VC, et al. Fluctuations of HIV load in semen of HIV positive patients with newly acquired sexually transmitted diseases. BMJ 1996:313:341-2.

21 Zhu T, Wang N, Carr A, et al. Genetic characterization of human immunodeficiency virus type 1 in blood and genital secretions: evidence for viral compartmentalization and selection during sexual transmission. J Virol 1996;70:3098-107.

22 Ball JK, Curran R, Irving WL, et al. HIV-1 in semen: determination of proviral and viral titres compared to blood, and quantification of semen leukocyte populations. J Med Virol 1999;59:356-63.

23 Paranipe S, Craigo J, Patterson B, et al. Subcompartmentalization of HIV-1 quasispecies between seminal cells and seminal plasma indicates their origin in distinct genital tissues. AIDS Res Human Retrovir 2002;18:1271-80.

24 Ping LH, Cohen MS, Hoffman I, et al. Effects of genital tract inflammation on human immunodeficiency virus type $1 \mathrm{~V} 3$ populations in blood and semen. $J$ Virol 2000;74:8946-52.

25 Chakraborty $\mathbf{H}$, Sen PK, Helms RW, et al. Viral burden in genital secretions determines male-to-female sexual transmission of HIV-1: a probabilistic empiric model. AIDS 2001;15:621-7.

26 Varela JA, Otero L, Garcia MJ, et al. Trends in the prevalence of pathogens causing urethritis in Asturias, Spain, 1989-2000. Sex Transm Dis 2003;30:280-3.

27 Benn P, Rooney G, Brown M, et al. The performance of the ligase chain reaction (LCR) in determining the prevalence of pharyngeal, urethral and rectal Chlamydia trachomatis and Neisseria gonorrhoeae in homosexua men. Berlin: ISSTDR, 2001

28 Hobbs MM, Kazembe P, Reed AW, et al. Trichomonas vaginalis as a cause of urethritis in Malawian men. Sex Transm Dis 1999;26:381-7.

29 Wendel KA, Erbelding EJ, Gaydos CA, et al. Use of urine polymerase chain reaction to define the prevalence and clinical presentation of Trichomonas vaginalis in men attending an STD clinic. Sex Transm Dis 2003;79:151-3.

30 Taylor S, Sadiq ST, Weller I, et al. Drug-resistant HIV-1 in the semen of men receiving antiretroviral therapy with acute sexually transmitted infections. Antivir Ther 2003;8:479-83. 